\title{
Sleep Apnoeas
}

\section{John M Shneerson}

\section{Introduction}

Sleep apnoeas are common but only became generally recognised in the 1980's. The rise in awareness of the condition among the general public, general practitioners and other healthcare professionals is rapidly increasing the number of patients who are being diagnosed and requiring treatment.

A sleep apnoea is conventionally diagnosed as the cessation of airflow for ten or more seconds. There are two main types of apnoea, central and obstructive. In central apnoeas there is no apparent movement of the rib cage or abdomen. This may be due either to an absence of the respiratory drive or to extreme weakness of the respiratory muscles so that the drive to breathe cannot be translated into movement. The more common obstructive sleep apnoeas are, in contrast, the result of transient obstruction of the upper airway during sleep. Respiratory efforts continue, but air cannot enter the lungs. The increasing efforts of the chest wall muscles are the main stimulus to arouse from sleep, and at this moment there is a loud snorting or gasping noise. The subject may jerk or jolt awake, although there is usually no recollection of the arousal. Deep inspirations after the apnoea rapidly normalise the arterial $\mathrm{pO}_{2}$ and $\mathrm{pCO}_{2}$. The subject then falls asleep again and the cycle is repeated, even up to 500 times each night.

\section{Central Sleep Apnoeas (CSA)}

These may occur in several different situations

\section{Normal Subjects}

It is common for occasional CSAs to occur during rapid eye movement (REM) sleep in which the respiratory pattern can be very erratic. Occasionally normal subjects also show CSAs at the moments of transition from lighter to deeper stages of non rapid eye movement (NREM) sleep as the respiratory drive temporarily changes.

\section{Cheyne Stokes Respiration}

This pattern is characterised by regular waxing and waning of the tidal volume with little change in respiratory frequency. The intervals between the deep breaths usually exceed ten seconds and are therefore CSAs. Cheyne Stokes Respiration is due to an instability of the respiratory drive which is often associated with a slow circulation time, usually due to cardiac failure or other low cardiac output states. It can also be due to dysfunction of the cerebral cortex which influences the reflex control of respiration.

Treatment of the underlying cause is important but if the Cheyne Stokes Respiration persists, and particularly if it causes arousals from sleep it is often effectively treated with oxygen or a nasal continuous positive airway pressure (CPAP) system (see below).

\section{Neuromuscular and Skeletal Disorders}

These disorders individually are uncommon, but together comprise an important group of conditions which cause CSAs leading to respiratory failure with a high arterial $\mathrm{pCO}_{2}$. Occasionally the respiratory centres themselves are damaged, as in central alveolar hypoventilation, but more commonly there is either weakness of the respiratory muscles as in for instance, muscular dystrophies, previous poliomyelitis or motor neurone disease, or an obvious disorder of the thoracic skeleton, such as a scoliosis, kyphosis, or following a thoracoplasty for treatment of previous tuberculosis. If sleep apnoeas are suspected in any of these disorders referral for an urgent specialist opinion is required since without treatment the prognosis is poor. Non invasive nocturnal ventilation has transformed the outlook for these patients and is usually required each night in the long term.

\section{Obstructive Sleep Apnoeas (OSA)}

\section{What Causes OSA?}

Whether or not the upper airway closes during sleep depends on the balance of forces between the muscles tending to hold the airway open (dilators) and the forces working to constrict it. The two most important of these are the negative pressure in the airway during inspiration and the mass of the tissues around the airway in the neck. With every inspiration the negative pressure generated inside the chest by the diaphragm and other chest wall muscles is transmitted through the upper airway to the mouth and nose. This tends to suck the walls of the airway together, and this is encouraged by the weight of the tissues, including fatty tissue, in the neck. The extent to which the dilator muscles in the upper airway are able to counteract these forces varies from person to person and this may be one of the major factors determining why some subjects develop OSA and others do not. Any lesion between the larynx and the soft palate that narrows the airway, such as large tonsils, also predisposes to the airway closing during sleep and OSA appearing.

\section{Who gets OSA?}

$80 \%$ of subjects with OSA remain undiagnosed and untreated. The condition is four times commoner in males than females and affects up to $4 \%$ of the adult male population. Sleep apnoeas can also occur in children, particularly if there is a congenital craniofacial abnormality (eg, Pierre-Robin syndrome) or if they have large tonsils or adenoids. In adults the most common cause is obesity. Fat is laid down in the neck to a greater extent in men than in premenopausal women, but individual variations in skeletal and soft tissue morphology may also 


\section{Not to be reproduced without the permission of the Primary Care Respiratory Journal}

determine which subjects are likely to develop OSA. Micrognathia or a posteriorly positioned mandible (retrognathia) also predispose to OSA, particularly when the subject lies in the supine position and the tongue falls back into the narrowed pharynx. Neuromuscular conditions such as previous poliomyelitis or motor neurone disease also occasionally weaken the dilator muscles in the pharynx and lead to OSA.

\section{How can OSA be diagnosed?}

A careful history is essential to diagnose sleep apnoeas. The cardinal symptoms are snoring and snorting at night, often with the partner's observation of stopping breathing or struggling to breathe, plus waking feeling unrefreshed in the morning and falling asleep readily during the day. The daytime sleepiness is due to fragmentation of sleep by the frequent arousals at night. Other common symptoms include nocturnal choking or breathlessness, nocturia, restlessness during sleep, irritability, and poor memory and concentration during the day.

In children the sleep fragmentation due to sleep apnoeas often causes irritability, hyperactivity and aggression similar to that seen in the attention deficit hyperactivity disorder (ADHD). Poor school performance and learning problems are common. There may also be developmental delay with short stature probably due to a reduction in secretion of growth hormone due to loss and fragmentation of stages 3 and 4 NREM sleep.

Risk factors such as obesity, large tonsils, retrognathia or micrognathia should be sought and the diagnosis can be confirmed by a sleep study. An oximeter which continuously records both the oxygen saturation and heart rate may be sufficient, but the trace needs careful interpretation and should be assessed in the context of the clinical features. More complex studies involving measurement of the airflow at the mouth and nose, chest and abdominal movement and the recording of sounds made during sleep may be needed. Polysomnography in which the electroencephalogram, electrooculogram and electromyogram are also recorded is rarely needed unless other conditions such as narcolepsy are being considered.

\section{Does OSA matter?}

The effects on sleep and wakefulness of OSA often have important implications for the quality of life of both the patient and partner. Marital problems, loss of employment and difficulty in interpersonal relationships are common. The risk of road traffic accidents is increased around six times in those with severe OSA and is due to falling asleep at the wheel. This often causes single vehicle events with a higher risk of fatalities than in non sleep-related accidents. They tend to occur between 2.00-6.00am and 2.00$4.00 \mathrm{pm}$ which is when the circadian rhythms favour sleepiness and magnify the sleepiness due to OSA.

During each apnoea the heart rate and blood pressure fluctuate with the latter often increasing by $50 \%$ during each episode. These surges in blood pressure together with changes in clotting are thought to underly the three times increased risk of stroke in those with severe OSA. Myocardial infarction and hypertension during wakefulness are also more common than in normal subjects, possibly due to the sustained sympathetic drive throughout the day associated with poor quality sleep at night. The repetitive dips of oxygen saturation can eventually lead to chronic respiratory failure with hypercapnia and right heart failure.

\section{What can the general practitioner do?}

OSA should be considered by the GP in any patient with a history of snoring and daytime sleepiness. Sleepiness should be distinguished from 'tiredness' which may mean physical weariness or mental fatigue rather than true sleepiness. The Epworth Sleepiness Score (Fig 1) is a quick and useful means of assessing whether or not the patient is sleepy. The normal value is less than 11 .

Once the GP has suspected the diagnosis there are two possible courses of action. If it is felt that the apnoeas are mild it may be sufficient to provide first line treatment (see below), but if they are more serious, and particularly if they affect the ability to drive or work, referral to a specialist centre for a sleep study to confirm the diagnosis should be made.

\section{Figure 1: EPWORTH SLEEPINESS SCALE (ESS)}

How likely are you to fall asleep in the following situations, in contrast to just feeling tired?

This refers to your usual way of life in the last few weeks. Even if you have not done some of these things recently, try to work out how they would have affected you. Use the following scale to choose the most appropriate number for each situation.

$0=$ Would never doze
$2=$ Moderate chance of dozing

Situation

Sitting and reading

Watching TV

Sitting inactive in a public place, (eg, a theatre or a meeting)

As a passenger in a car for an hour without a break

Lying down in the afternoon, when circumstances allow

Sitting and talking to someone Sitting quietly after lunch without alcohol

In a car, while stopped for a few minutes in traffic

$$
\begin{aligned}
& 1=\text { Slight chance } \\
& 3=\text { High chance of dozing }
\end{aligned}
$$

1 3

$\begin{array}{llll}0 & 1 & 2 & 3\end{array}$

$\begin{array}{llll}0 & 1 & 2 & 3\end{array}$

$\begin{array}{llll}0 & 1 & 2 & 3\end{array}$

$\begin{array}{llll}0 & 1 & 2 & 3\end{array}$

$\begin{array}{llll}0 & 1 & 2 & 3\end{array}$

$\begin{array}{llll}0 & 1 & 2 & 3\end{array}$

$\begin{array}{llll}0 & 1 & 2 & 3\end{array}$

$\begin{array}{llll}0 & 1 & 2 & 3\end{array}$




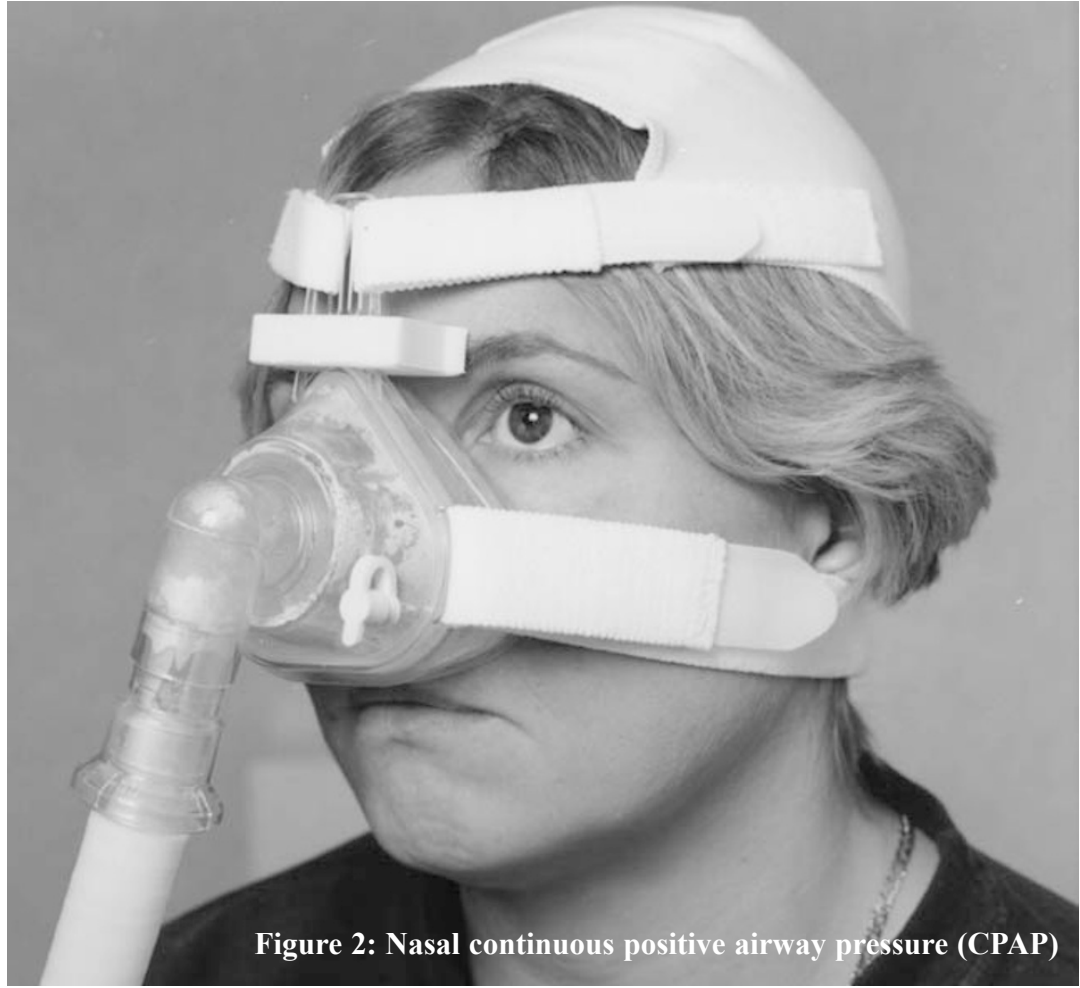

The most important first line treatments that the GP may initiate are

1. Weight loss - This should be attempted in all patients who are obese, particularly if weight gain has coincided with an increase in neck size and a worsening of symptoms.

2. Reduction in alcohol intake

3. Cessation of smoking

4. Avoidance of benzodiazepines and other hypnotics

5. Sleep hygiene - Sleep deprivation exacerbates sleep apnoeas.

6. Advise sleeping on the side rather than in the supine position - Postural apnoeas are common and sleeping with a pillow wedged under the shoulders to prevent returning to the supine position is often effective.

7. Improvement in nasal airway, eg, treatment with inhaled steroids

\section{What else can be done for OSA?}

If the subject's apnoeas remain troublesome despite first line treatments the other more specialist options are

1. Surgery - Uvulopalatopharyngoplasty (UPPP) is effective in $60-70 \%$ of non obese subjects who snore, but it is of little value in those with sleep apnoeas.

Nasal surgery is of no benefit in sleep apnoeas, but removal of enlarged tonsils is often curative.

Tracheostomy is effective since it bypasses the upper airway obstruction, but is rarely required.

2. Mandibular advancement devices - These hold the mandible forward so that the tongue cannot fall back into the pharyngeal airway during sleep. Many of the devices are in a single piece, but others are hinged. Some can be moulded by the patient, but others are designed individually by a dental surgeon from an impression of the teeth.

These devices are most effective if the apnoeas originate at the level of the base of the tongue, if they are worse in the supine position and if the subject is not obese and if the apnoeas are only mild to moderately severe. They are contraindicated if there is temporo-mandibular joint dysfunction, insufficient or excessively mobile anterior teeth, or dentures which are removed at night.

3. Nasal continuous positive airway pressure (CPAP). The principle of nasal CPAP (Fig 2) is that it increases the pressure within the upper airway both during inspiration and expiration. It acts as a pneumatic splint counteracting the forces that tend to close the airway. A pump with a high flow capacity delivers air at a pressure of usually $5-15 \mathrm{~cm} \mathrm{H}_{2} 0$, but which can be adjusted to suit the patient individually. Some modern machines vary the pressure during the night, but these have little advantage over the standard equipment. The pump connects to the mask by tubing which has a leak to prevent rebreathing of carbon dioxide. A nasal mask is usually preferable to a full face mask which includes the mouth as well. The masks are usually made of silicone or gel and are held in place by headgear so that they fit snugly on the face.

Nasal CPAP is usually well tolerated but can cause ulcers over the bridge of the nose, air leaks around the mask, airway symptoms particularly nasal blockage, and claustrophobia. Nasal symptoms may be improved with an inhaled nasal steroid or a humidifier inserted into the circuit.

It is important to educate the patient in how to use nasal CPAP. Regular supervision is needed in order to ensure that problems are overcome and that the CPAP level remains effective. Compliance with CPAP is usually around $75 \%$ and in some centres up to $90 \%$. CPAP is able to reverse the excessive daytime sleepiness and physiological abnormalities of OSA, but these benefits are lost as soon as treatment is discontinued.

\section{Modafinil}

This new non amphetamine wake promoting drug has been proposed as treatment to relieve daytime sleepiness associated with sleep apnoeas if this persists despite adequate treatment with nasal CPAP. Its place has yet to be established.

\section{Further Reading}

1. Shneerson JM, Handbook of Sleep Medicine. Blackwell Science, Oxford 2000.

2. Stradling JR, Handbook of Sleep-Related Breathing Disorders, Oxford Medical Publications 1995. 